\title{
Quantitative proteomics reveals docosahexaenoic acid-mediated neuroprotective effects in lipopolysaccharide-stimulated microglial cells
}

Bo Yang ${ }^{1,3}$, Runting Li ${ }^{2}$, Pei N. Liu ${ }^{3}$, Xue Geng ${ }^{4}$, Brian P. Mooney ${ }^{2,3}$, Chen Chen ${ }^{5}$, Jianlin Cheng $^{5}$, Kevin L. Fritsche ${ }^{6}$, David Q. Beversdorf ${ }^{7}$, James C. Lee ${ }^{4}$, Grace Y. Sun ${ }^{2}$ and C. Michael Greenlief 1,3*

${ }^{1}$ Department of Chemistry, ${ }^{2}$ Biochemistry Department, ${ }^{3}$ Charles W. Gehrke Proteomics Center, ${ }^{5}$ Department of Electrical Engineering and Computer Science, ${ }^{6}$ Department of Nutrition and Exercise Physiology and ${ }^{7}$ Departments of Radiology, Neurology and Psychological Sciences, and the Thompson Center, University of Missouri, Columbia, MO, USA; ${ }^{4}$ Department of Bioengineering, University of Illinois at Chicago, IL, USA.

\section{$\underline{\text { Supplemental Material }}$}

Supplemental Material S1. Cell viability assayed by WST1 from two different cell passages. (page $\mathrm{S} 1$ )

Supplemental Material S2. Western blot images for Figure 5 in the manuscript (pages S2-S8) Supplemental Material S3. Table S1: Correlation coefficients for the three replicates of the four experimental groups. (page S9)

Supplemental Material S4. Excel file of the protein abundance data analyzed in R (separate Excel file)

\section{Supplemental Material S1. Figure S1: Cell viability assayed by WST1 from two} different cell passages.

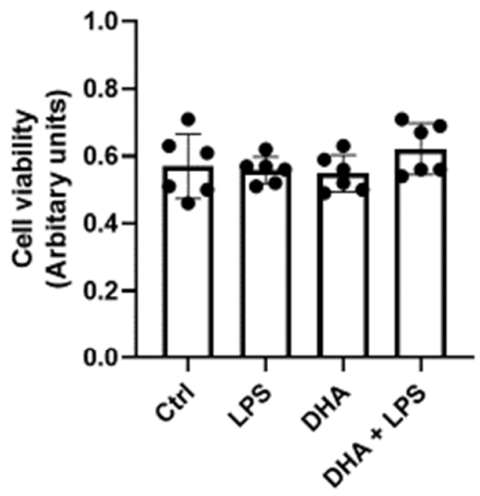

Cell viability assayed by WST1. Results are mean $+/-$ SD from two cell passages with each passage carried out in triplicates. 


\section{Supplemental Material S2. Western blot images for Figure 5.}

Western blots used to generate the images used in Figure 5 of the manuscript. Below are smaller pictures of the blots and descriptions. The name of the tiff file is below each image. The subsequent pages are the .tiff images with text.

Supplemental Material S2. Figure S1: p-p65 (control, DHA, LPS, DHA+LPS):

Below are two images of the same blot image taken under different exposure times for getting a good quality band. Lane 1 has trial 1 and 2, Lane 2 has trial 3 and 4 . The order of lanes from left to right is control, LPS treated, DHA treated, and DHA+LPS treated and the same order is repeated for the next four lanes.

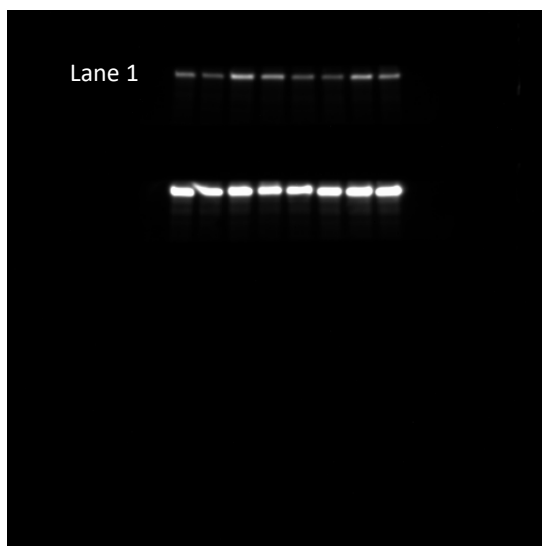

p-p65 Chemi 45s 20190326 1246.tiff

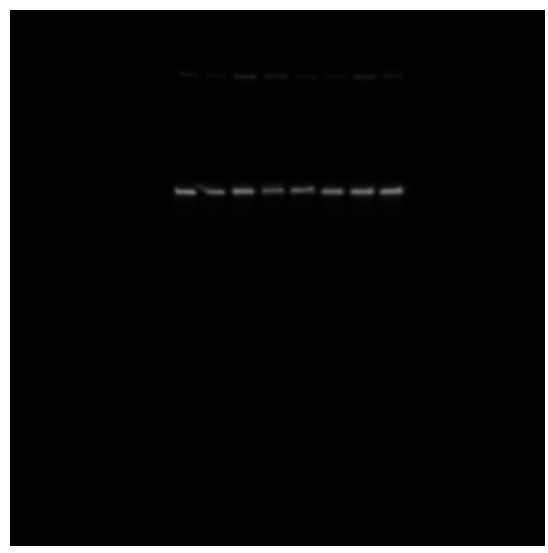

p-p65 Chemi 6s 20190326 1244. tiff

Supplemental Material S2. Figure S2: p-65 (control, DHA, LPS, DHA+LPS):

Lane 1 has trial 1 and 2, Lane 2 has trial 3 and 4 . The order of lanes from left to right is control, LPS treated, DHA treated, and DHA+LPS treated and the same order is repeated for the next four lanes.

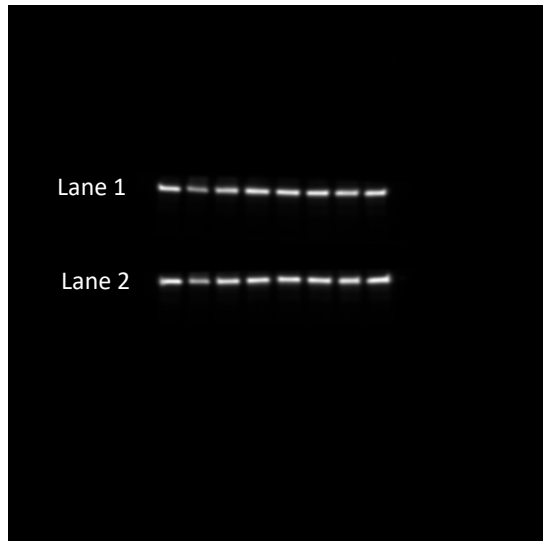

p65 Chemi 8s 20190326 1248.tiff 
Supplemental Material S2. Figure S3: iNOS (control, DHA, LPS, DHA+LPS):

Only used Lane 1 and Lane 2, the other two lanes are the replicates of Lane 1 and 2.

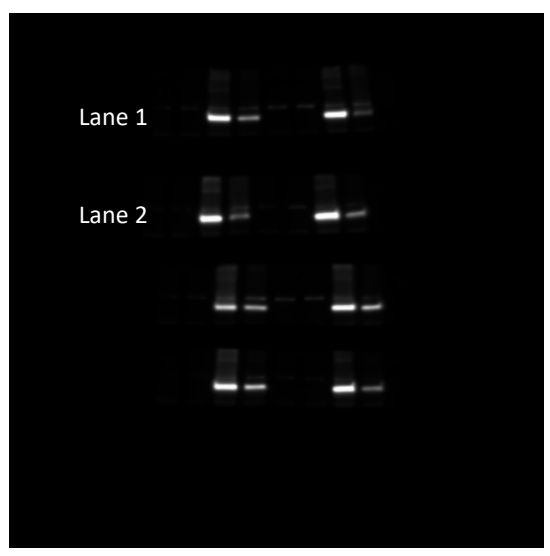

iNOS Chemi 8s 20190326 1254.tiff

Supplemental Material S2. Figure S4: $\beta$-actin (control, DHA, LPS, DHA+LPS):

Only used Lane 1 and Lane 2, the other two lanes are the replicates of Lane 1 and 2. The order of lanes from left to right is control, LPS treated, DHA treated, and DHA+LPS treated and the same order is repeated for the next four lanes.

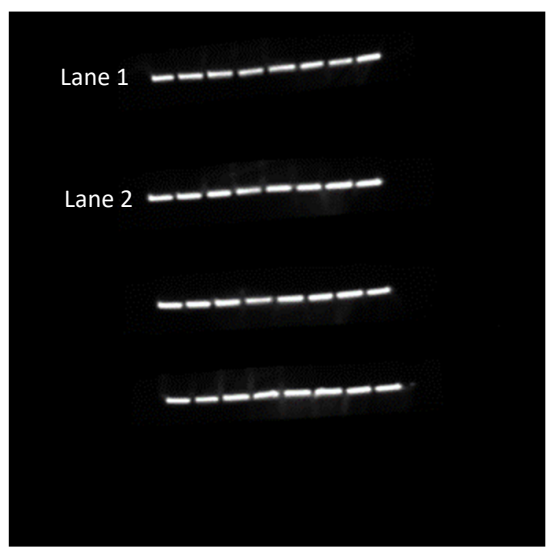

beta-actin Chemi 16s 20190327 1132.tiff 


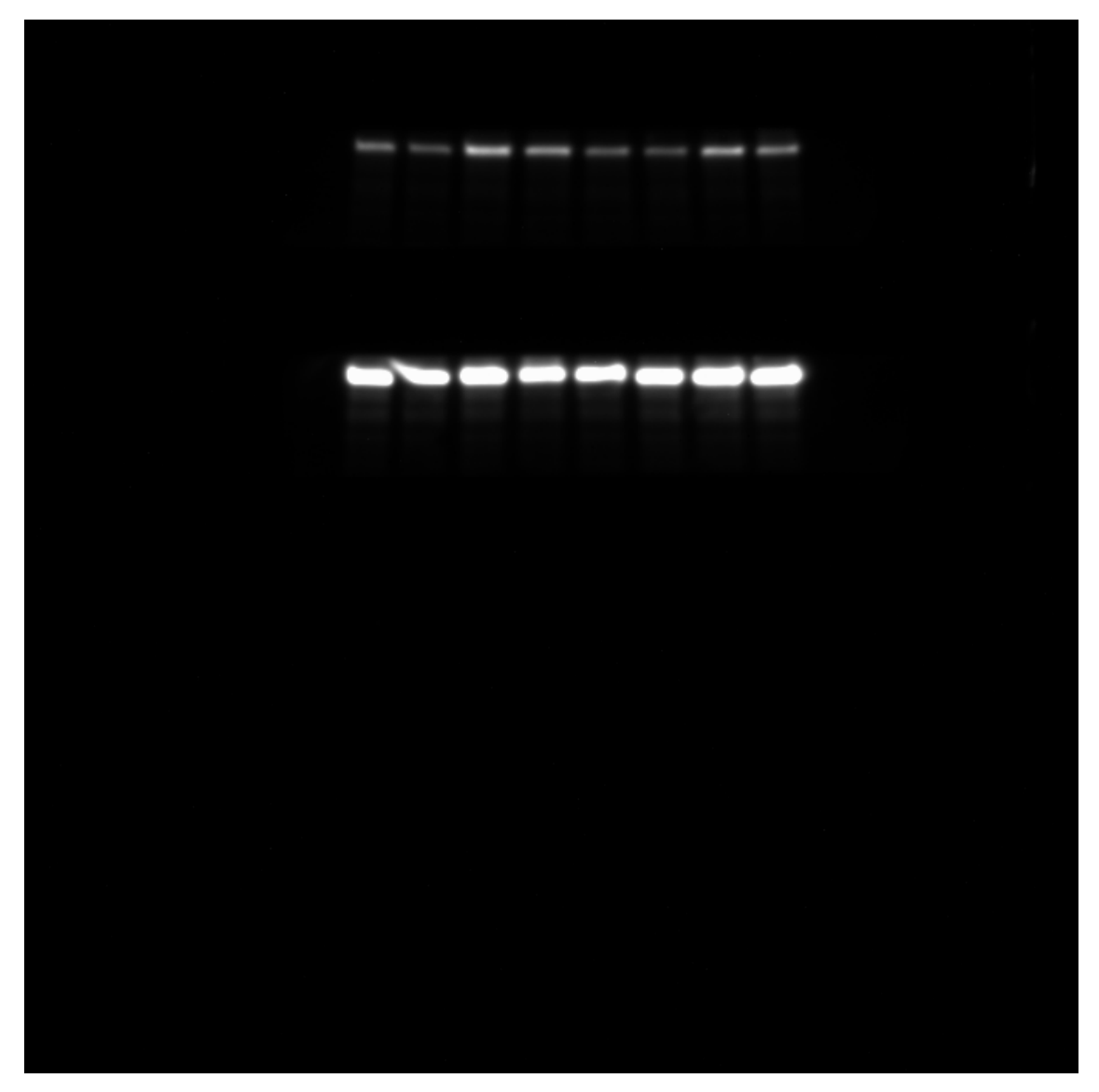

Supplemental Material S2. Figure S5: p-p65 Chemi 45s 20190326 1246.tiff 


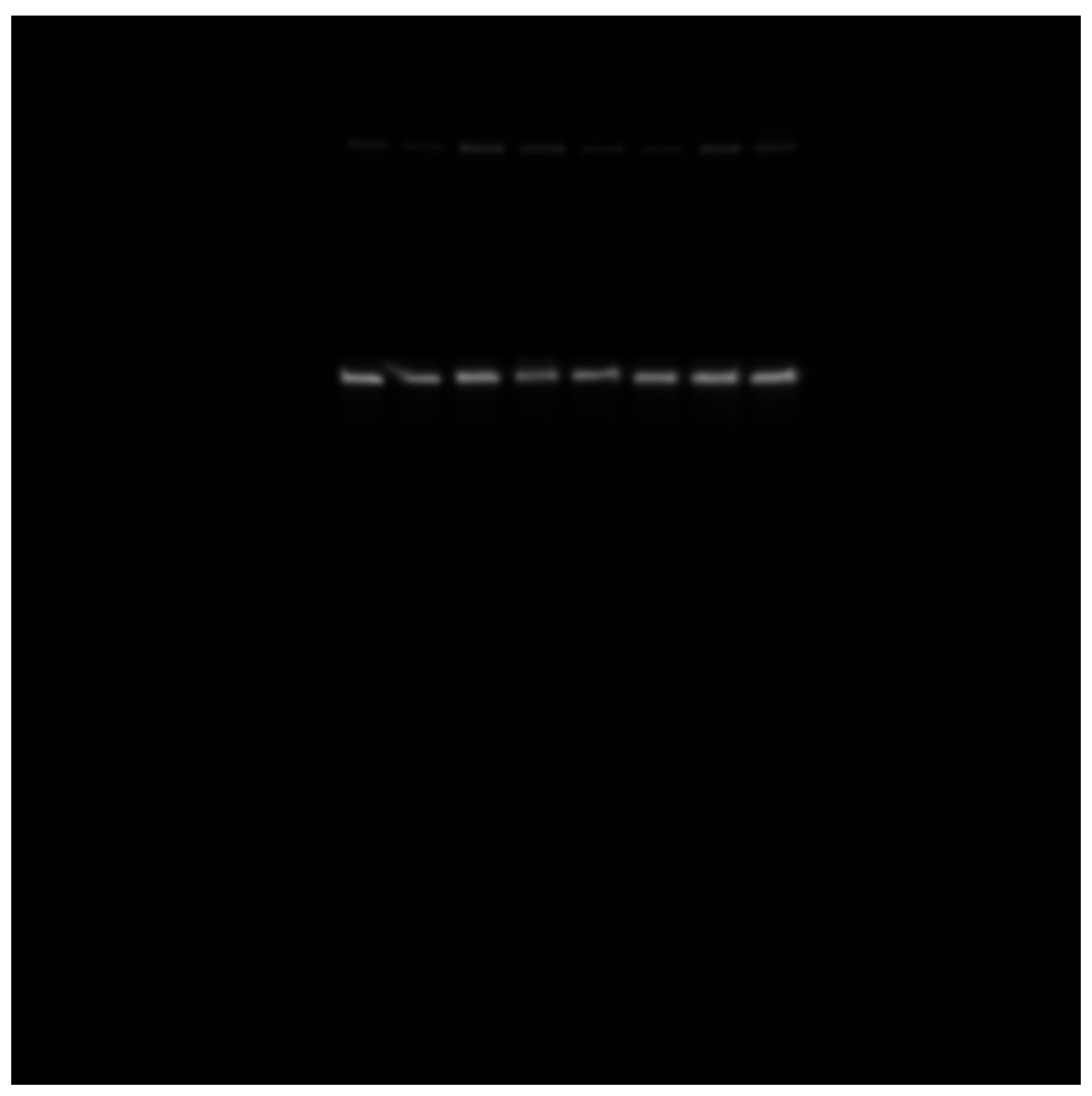

Supplemental Material S2. Figure S6: p-p65 Chemi 6s 20190326 1244. Tiff 


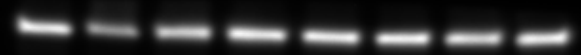

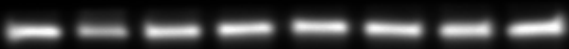

Supplemental Material S2. Figure S7: p65 Chemi 8s 20190326 1248.tiff 


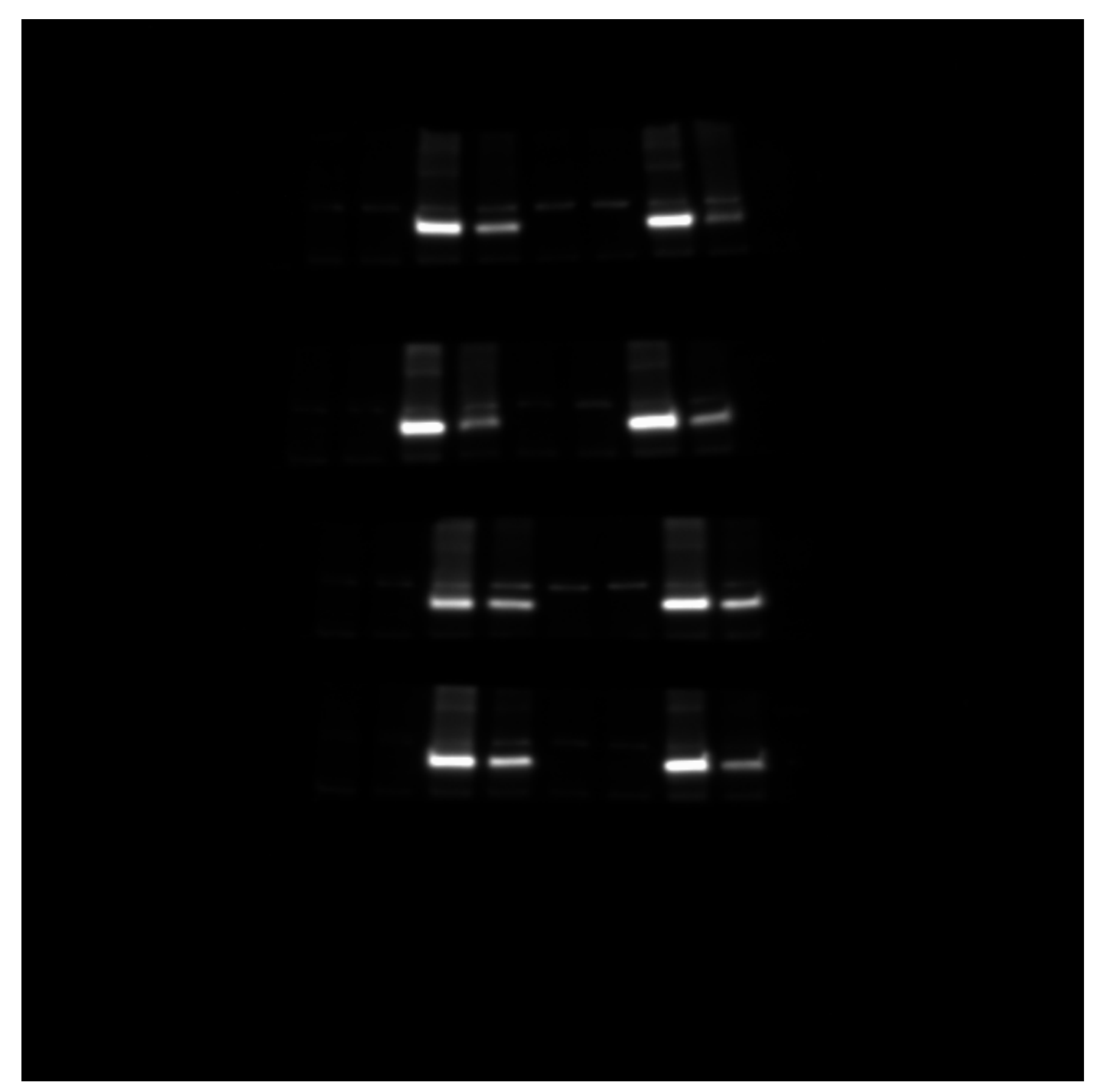

Supplemental Material S2. Figure S8: iNOS Chemi 8s 20190326 1254.tiff 


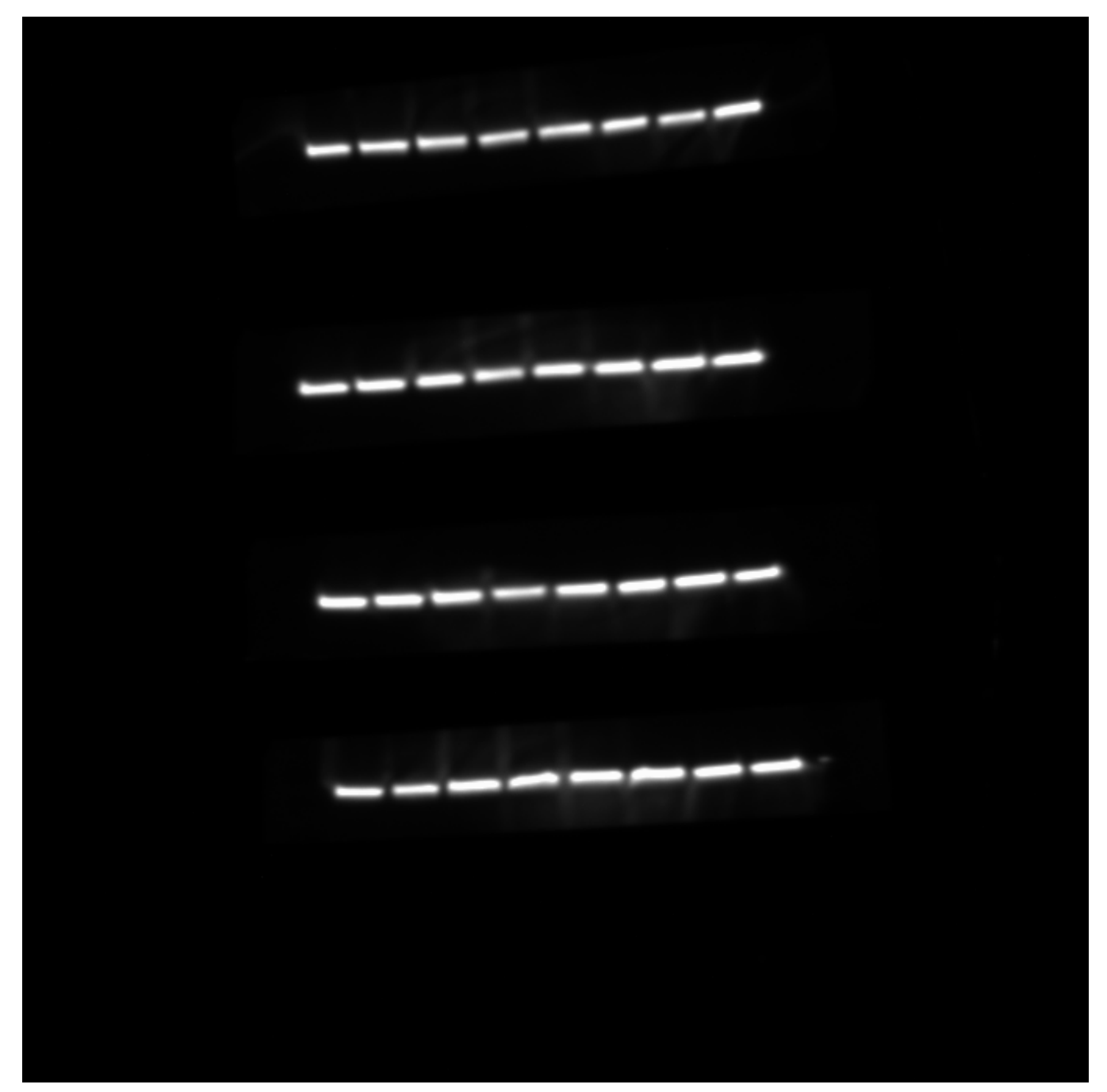

Supplemental Material S2. Figure S9: beta-actin Chemi 16s 20190327 1132.tiff 


\section{Supplemental Material S3. Correlation coefficients of the three replicates for the four groups}

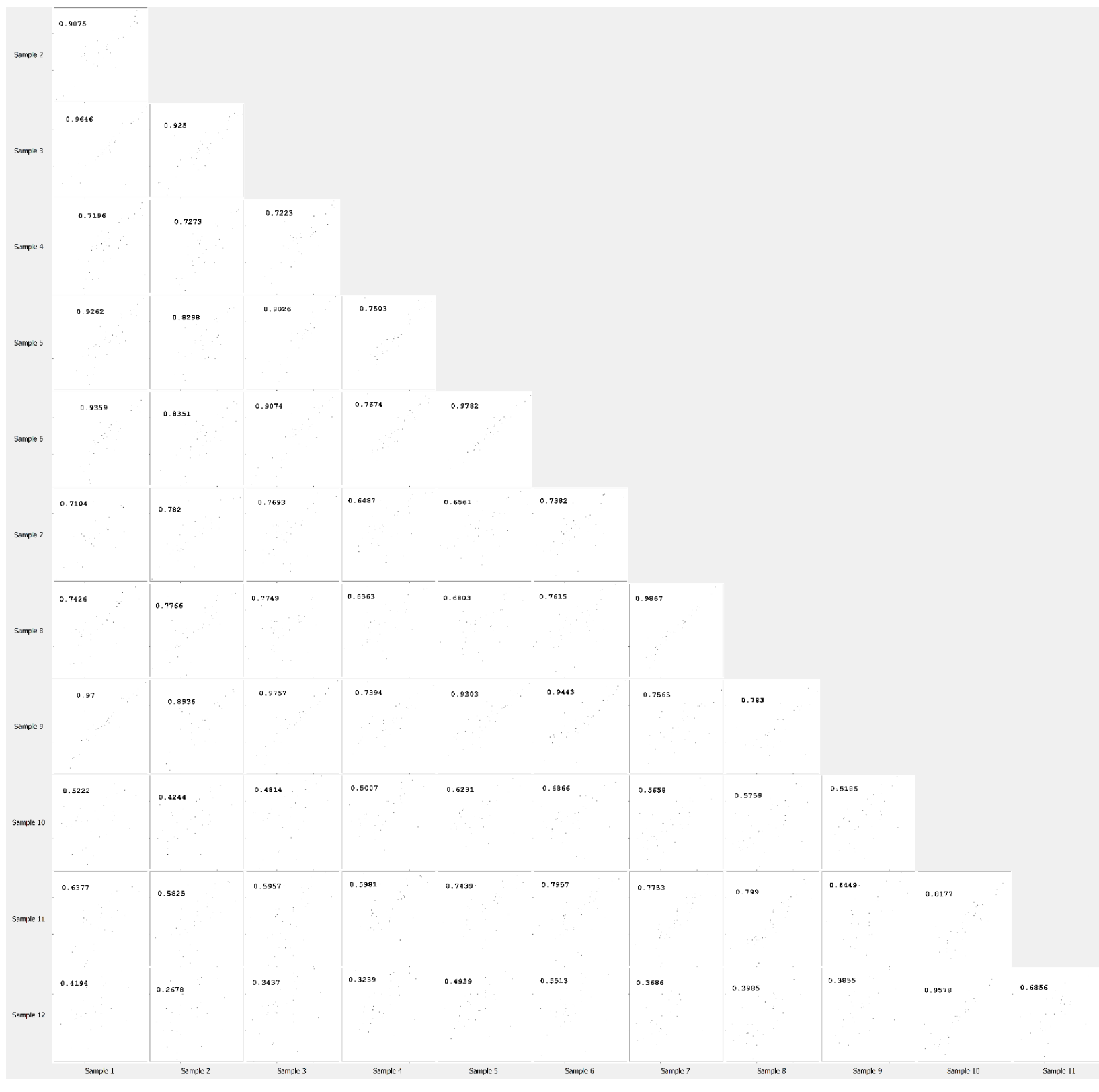

Supplemental Material S3. Table S1: Correlation coefficients of the three replicates for the four groups. Samples 1-3, cells are under normal condition; Samples 4-6, cells are treated with LPS; Samples 7-9, cells are treated with DHA; Samples 10-12, cells are pretreated with DHA followed with LPS. 